\title{
Índice de éxito y morbilidad de la nefrolitotomía percutánea
}

\section{Success and morbidity rates in patients treated with percutaneous nephrolithotomy}

Karian Trujillo-Ríos, ${ }^{1}$ Rodolfo Galeana-Ruiz, ${ }^{2}$ Felipe de Jesus González-González, ${ }^{3}$ Román Carvajal-García, ${ }^{4}$ Ernesto Mendoza-Villanueva, ${ }^{5}$ Mario Beltrán-de la Peña, ${ }^{6}$ Iván Emmanuel Michel-Mercado ${ }^{6}$

\begin{abstract}
Resumen
OBJETIVO: Determinar la tasa de éxito y las complicaciones relacionadas con la nefrolitotomía percutánea.

MATERIALES Y MÉTODOS: Estudio transversal y analítico, efectuado en pacientes intervenidos de nefrolitotomía percutánea, atendidos en el Hospital Regional Dr. Valentín Gómez Farías, entre marzo de 2014 y octubre de 2017. Se analizaron variables como: edad, sexo, riñón afectado, antecedente de cirugía renal o litotricia extracorpórea y comorbilidades asociadas, además de las características, localización y microbiología de los cálculos renales; la tasa libre de cálculos y complicaciones. Para el análisis de los datos se utilizó el programa estadístico SPSS, versión 10; se implementó la prueba exacta de Fisher y se consideró estadísticamente significativo el valor de $\mathrm{p}<0.05$.

RESULTADOS: Se incluyeron 191 pacientes: $41.8 \%$ correspondió a hombres y $58.1 \%$ a mujeres, con edad promedio de $56.9 \pm 12.1$ años. El riñón derecho fue el más afectado (52\%). El $36 \%$ de los pacientes tenía antecedente de cirugía renal y $22 \%$ había recibido litotricia con ondas de choque. De igual forma, $36 \%$ padecía algún tipo de comorbilidad. El diámetro promedio de los cálculos fue de $2.52 \mathrm{~cm}$ y volumen de 2.98 cc. La localización más frecuente fue el cáliz inferior, seguida de la pelvis renal. El 29\% de los cultivos fueron positivos y Escherichia coli, Proteus mirabilis, Klebsiella pneumoniae y Pseudomonas aeruginosa fueron los microorganismos más frecuentes. La tasa libre de cálculos fue de $78 \%$ y de complicaciones $18 \%$.

CONCLUSIONES: La nefrolitotomía percutánea es una técnica efectiva en el tratamiento de la litiasis renal, incluso disminuye la tasa de complicaciones.
\end{abstract}

PALABRAS CLAVES: Nefrolitotomía percutánea; complicaciones; tasa libre de cálculos.

Abstract

OBJECTIVE: To determine the success rate and complications related to percutaneous nephrolithotomy.

MATERIALS AND METHODS: An analytic, cross-sectional study was conducted on patients that underwent percutaneous nephrolithotomy at the Hospital Regional Dr. Valentín Gómez Farías, within the time frame of March 2014 to October 2017. The variables analyzed were: age, sex, affected kidney, history of kidney surgery or extracorporeal lithotripsy, associated comorbidities, kidney stone characteristics, location and microbiology, stone-free rate, and complications. The data analysis was carried out using the SPSS version 10 statistics program. The Fisher's exact test was employed and statistical significance was set at a $\mathrm{p}<0.05$.

RESULTS: A total of 191 patients were included, $41.8 \%$ of whom were men and $58.1 \%$ women. The mean age was $56.9 \pm 12.1$ years. The right kidney was the most affected $(52 \%)$. Thirty-six percent of the patients had a history of kidney surgery and $22 \%$ had undergone shock wave lithotripsy. Likewise, $36 \%$ presented with a comorbidity. Mean
${ }^{1}$ Residente del curso de alta especialidad en Endourología.

${ }^{2}$ Profesor titular del servicio de Endourología.

${ }^{3}$ Profesor adjunto del servicio de Endourología.

${ }^{4}$ Jefe del servicio de Urología.

${ }^{5}$ Profesor adjunto del servicio de Urología.

${ }^{6}$ Residente de Urología.

Hospital Regional Dr. Valentín Gómez Farías (ISSSTE), Zapopan, Jalisco.

Recibido: julio 2018

Aceptado: noviembre 2018

Correspondencia Karian Trujillo Rios

dr.katrujillo@gmail.com

Este artículo debe citarse como Trujillo-Ríos K, Galeana-Ruiz R, González-González FJ, Carvajal-García $\mathrm{R}$ Mendoza-Villanueva E, Beltrán-de la Peña M, Michel-Mercado IE. Índice de éxito y morbilidad de la nefrolitotomía percutánea. Rev Mex Urol. 2018 novdic;78(6):434-39.

DOI: https://doi.org/10.24245/revmexurol.v78i6.2398 
stone diameter was $2.52 \mathrm{~cm}$ and mean volume was $2.98 \mathrm{cc}$. The most frequent location was the lower calyx, followed by the renal pelvis. Twenty-nine percent of the cultures were positive. The most frequent microorganisms were Escherichia coli, Proteus mirabilis, Klebsiella pneumoniae, and Pseudomonas aeruginosa. The stone-free rate was $78 \%$ and the complication rate was $18 \%$.

CONCLUSIONS: Percutaneous nephrolithotomy is an effective technique in the treatment of kidney stones, reducing the complication rate.

KEYWORDS: Percutaneous nephrolithotomy; Complications; Stone-free rate.

\section{ANTECEDENTES}

La litiasis urinaria es una alteración caracterizada por cálculos en el aparato genitourinario. Representa una de las enfermedades más comunes en la sociedad moderna. Los factores de riesgo asociados incluyen: edad, sexo, ocupación, componentes genéticos, peso, consumo de líquidos, clima y área geográfica. ' La prevalencia de litiasis varía de $1-15 \%$, de acuerdo con la edad, raza, sexo y localización geográfica. En occidente la prevalencia ha sido estimada en $10-15 \% .^{2,3}$

En las últimas tres década, el avance en los métodos de mínima invasión ha facilitado la remoción de cálculos renales. ${ }^{2,3}$

Fernstrom y Johansson reportaron el primer procedimiento de remoción de un cálculo renal utilizando un tracto de nefrostomía. Posteriormente, la nefrolitotomía percutánea emergió como una técnica de rutina para el tratamiento de pacientes con cálculos mayores de $2 \mathrm{~cm}$ o cálculos complejos. ${ }^{4}$

Los procedimientos percutáneos se asocian con menor morbilidad que la cirugía abierta; sin embargo, no están exentos de complicaciones. El reconocimiento y tratamiento oportuno de las complicaciones son cruciales, al igual que su prevención y disminución. ${ }^{5}$

Desde hace más de 20 años se utiliza el sistema de clasificación de Clavien, que se reevaluó y mo- dificó en 2004, con lo que incrementó su utilidad en múltiples áreas médicas, incluida la urología. ${ }^{6-9}$ Estudios recientes consideran el sistema Clavien modificado como el protocolo de referencia para clasificar las complicaciones ocurridas en la nefrolitotomía percutánea. ${ }^{7,9}$ Cuadro 1

Cuadro 1. Clasificación Clavien modificada ${ }^{8,9}$

\section{Grado Definición}

I Cualquier desviación del posoperatorio normal sin necesidad de tratamiento farmacológico, quirúrgico, endoscópico o radiológico intervencionista. Regímenes terapéuticos permitidos: fármacos (antieméticos, antipiréticos, analgésicos, diuréticos, electrolitos) y fisioterapia. Este grado también incluye infección de heridas abiertas en el paciente hospitalizado o en recuperación.

II Requerimiento de fármacos distintos a los permitidos para complicaciones de grado I. Trasfusiones sanguíneas y nutrición parenteral total.

III Intervención quirúrgica, endoscópica o radiológica.

IIla Procedimientos que no requieren anestesia general.

IIIb Intervenciones con anestesia general.

IV Complicación que pone en riesgo la vida del paciente y requiere su ingreso a la unidad de cuidados intensivos.

IVa Disfunción de un solo órgano (incluida la diálisis).

IVb Disfunción multiorgánica.

V Muerte del paciente.

Sufijo “d” El sufijo "d" (por disability) se anexa al grado de la complicación, en pacientes que requieren seguimiento después del alta hospitalaria, para su evaluación completa. 


\section{MATERIALES Y MÉTODOS}

Estudio transversal y analítico, efectuado en pacientes intervenidos de nefrolitotomía percutánea, atendidos en el Hospital Regional Dr. Valentín Gómez Farías entre marzo de 2014 y octubre de 2017. Criterios de inclusión: pacientes mayores de 18 años, atendidos en el servicio de Urología del hospital, operados de nefrolitotomía percutánea, con expediente clínico completo. Criterios de exclusión: pacientes que no cumplieron con los criterios mencionados. Se analizaron variables preoperatorias (edad, sexo, comorbilidades, localización del cálculo, determinación de hemoglobina, creatinina y urocultivo, y tratamientos previos), operatorias (tiempo quirúrgico, localización del cálculo y requerimiento de trasfusión sanguínea) y posoperatorias (complicaciones, exámenes de laboratorio [hemoglobina, creatinina] y litiasis residual).

Para el análisis de los datos se utilizó el programa estadístico SPSS versión 10. Se consideraron medidas de tendencia central (media, moda) y dispersión (desviación estándar, varianza y rango). Para la comparación entre variables de interés se implementó la prueba binomial para obtener el valor Z. Así mismo, se utilizó la prueba exacta de Fisher para variables cualitativas. Se consideró estadísticamente significativo el valor de $\mathrm{p}<0.05 \%$.

El estudio corresponde a nivel 2 de riesgo, según la Ley General de Salud, Título segundo de Los Aspectos Éticos de la Investigación en Seres Humanos, capítulo I, artículo 17. Es un estudio con riesgo mínimo; por lo tanto, no requiere consentimiento informado, pero se mantiene la confidencialidad de los datos de cada paciente. Fue aprobado por el comité de ética del Hospital Regional Dr. Valentín Gómez Farías.

\section{RESULTADOS}

Se incluyeron 191 pacientes tratados con nefrolitotomía percutánea por diagnóstico de litiasis renal: 80 (41.8\%) hombres y 111 (58.1\%) mujeres, con edad promedio de $56.9 \pm 12.16$ años.

En cuanto a las variables analizadas, el riñón más afectado fue el derecho en 99 (52\%) casos y el izquierdo en 92 (48\%). Sesenta y nueve (36\%) pacientes habían recibido algún tipo de tratamiento previo para la litiasis renal, ya sea pielolitotomía o nefrolitotomía anatrófica. Así mismo, a 42 (22\%) se les practicó litotricia extracorpórea por ondas de choque sin resultados satisfactorios.

Se registraron 68 (36\%) casos con algún tipo de comorbilidad, principalmente hipertensión arterial $18.3 \%(n=35)$ y diabetes mellitus $15.18 \%$ $(\mathrm{n}=29)$. Figura 1

El diámetro promedio de los cálculos renales fue de $2.54 \mathrm{~cm}$ y volumen de $2.98 \mathrm{cc}$. La localización renal más frecuente fue el cáliz inferior (39.2\%) y la pelvis renal (26.7\%). El 9.4\% de los casos correspondió a cálculos coraliformes completos, incluso algunos afectaban dos sitios. Figura 2

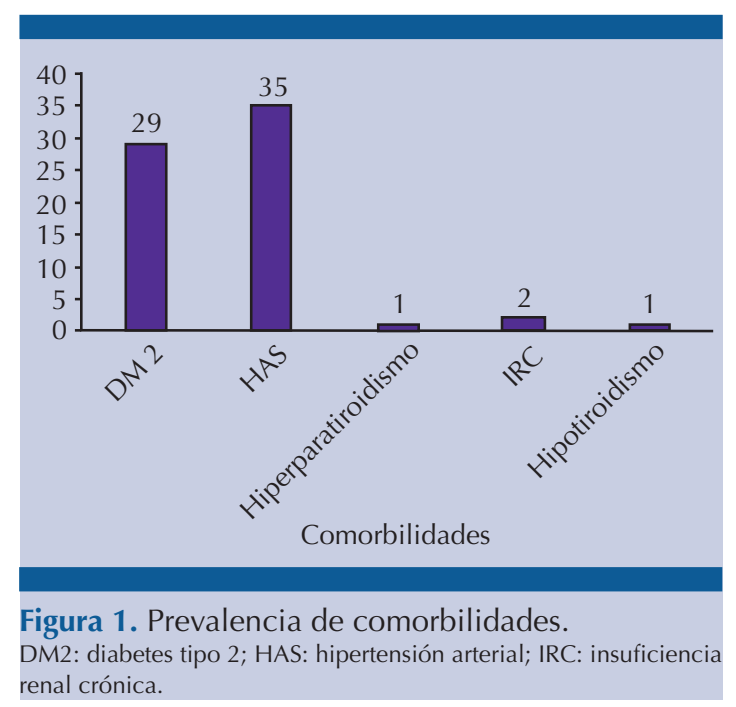




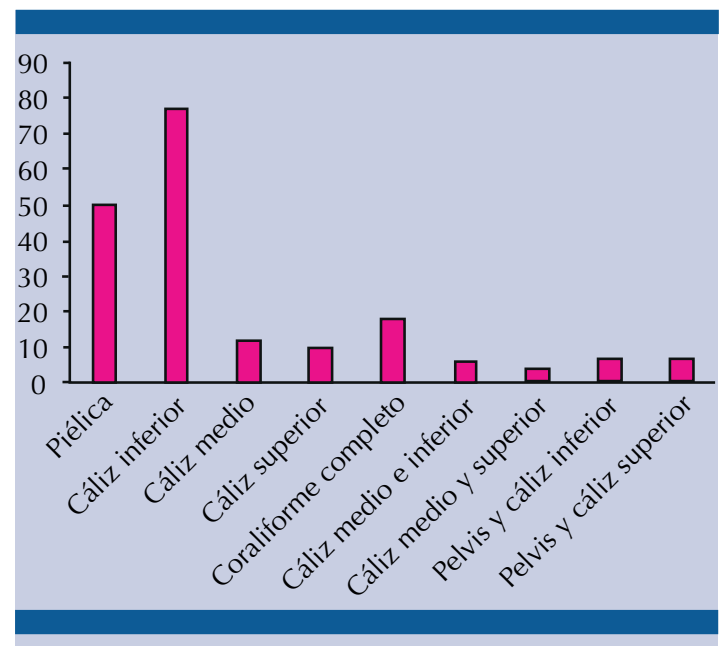

Figura 2. Localización de los cálculos renales.

El $29 \%$ de los casos $(n=56)$ tuvo cultivos de orina positivos. Escherichia coli fue el microorganismo más frecuente, seguido de Proteus mirabilis, Klebsiella y Pseudomonas aeruginosa (Figura 3). Se registró una tasa libre de cálculos de $78 \%$ ( $n=149)$, valorada mediante radiografía simple de abdomen (la definición más rigurosa y aceptada incluye: ausencia de cálculos o coexistencia de fragmentos menores de $2 \mathrm{~mm}) .^{10}$

Respecto de las complicaciones, se encontraron $34(18 \%)$ pacientes con algún tipo. Según la clasificación de complicaciones de la escala de Clavien modificada, 18 casos correspondieron

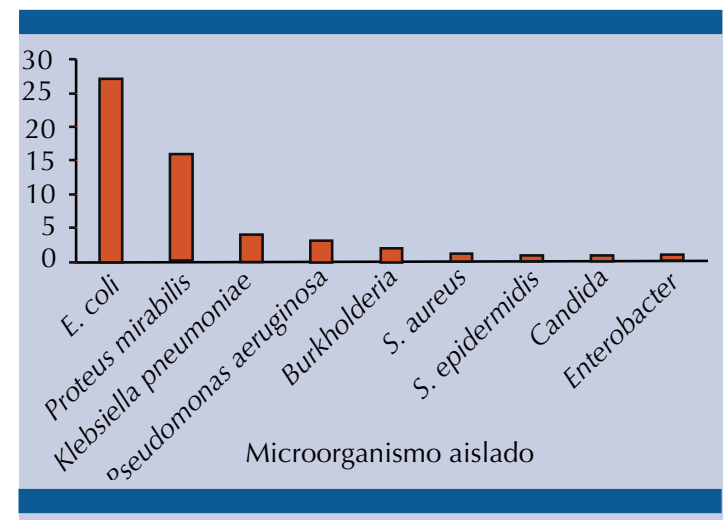

Figura 3. Microorganismos aislados del urocultivo. a Clavien I, 8 a Clavien II, 7 a Clavien IIla y 1 a Clavien IVa. El 82\% de los pacientes no tuvo complicaciones trans o posoperatorias. Figura 4

En cuanto a los exámenes de control, se observó disminución de la concentración de hemoglobina $(1.7 \mathrm{~g} / \mathrm{dL})$ e incremento de creatinina $(0.4 \mathrm{~g} / \mathrm{dL})$.

La tasa de éxito (libre de cálculos) de $78 \%$ en nuestro análisis fue discretamente superior a la reportada en el estudio Clinical Research Office of the Endourological Society (CROES) (75.7\%). Al aplicar la prueba exacta de Fisher se obtuvo un valor de 0.3512, por lo que no se observó significación estadística ( $p>0.05$ ). Las complicaciones registradas fueron similares a las reportadas en el estudio CROES, principalmente en las clasificaciones I, II y IIla. Según la prueba exacta de Fisher (valor de 0.4635), no se encontró diferencia estadísticamente significativa $(\mathrm{p}<$ 0.05). Figura 5

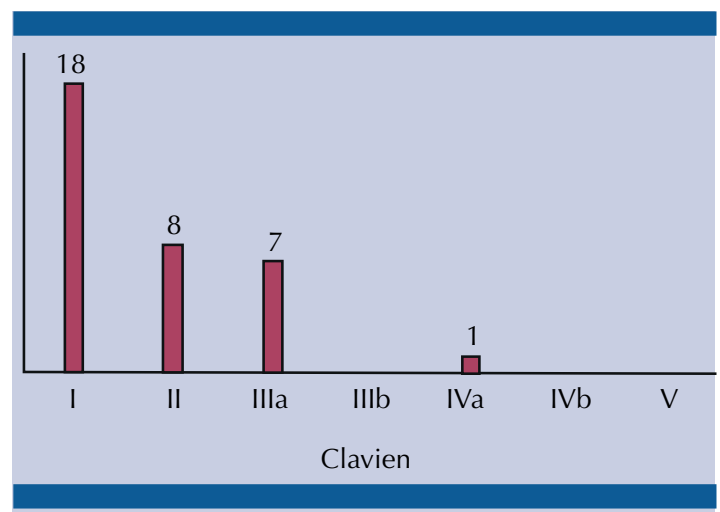

Figura 4. Complicaciones y clasificación de Clavien modificada.

\section{DISCUSIÓN}

Los procedimientos percutáneos renales se asocian con menor morbilidad que la cirugía abierta; sin embargo, no están exentos de complicaciones. Su reconocimiento y tratamiento oportuno es decisivo, igual que su prevención y disminución. ${ }^{5}$ 


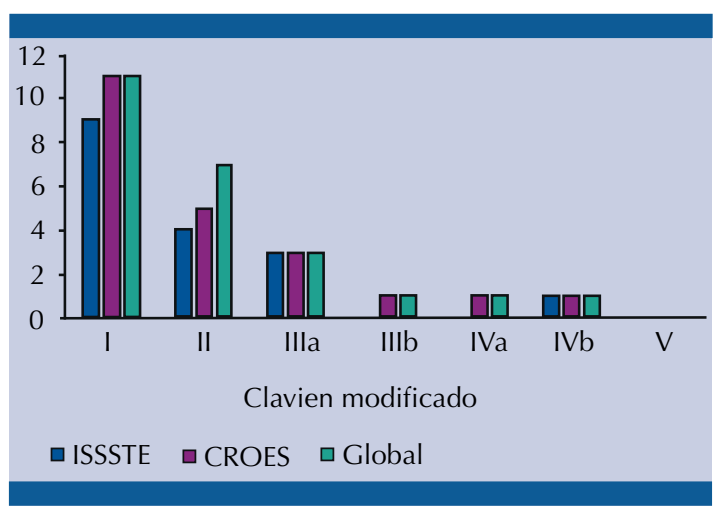

Figura 5. Comparación entre complicaciones, según la clasificación de Clavien modificado.

Este estudio de los índices de éxito y morbilidad representa la investigación más grande en pacientes con nefrolitotomía emprendida en nuestro hospital. Los resultados reflejan la variedad de indicaciones del procedimiento quirúrgico en pacientes con diversas características.

El primer análisis del estudio revela una tasa libre de cálculos de $78 \%$, que es comparable con $76 \%$ reportado en el CROES, el estudio con la base de datos más grande de pacientes tratados con nefrolitotomía percutánea. ${ }^{10-11}$

Los datos obtenidos revelan un índice de complicaciones de $18 \%$, documentadas con la clasificación de Clavien modificada, que demuestra ser un protocolo seguro. La hemorragia y las infecciones siguen siendo las principales complicaciones asociadas con la intervención y estas variables coincidieron con lo reportado en la bibliografía. ${ }^{7-10}$

Las tasas de éxito reportadas en algunos estudios se relacionan con características como: tamaño, volumen y localización del cálculo, índice de masa corporal y malformaciones genitourinarias o músculo-esqueléticas. ${ }^{7-9}$

La prevalencia baja de complicaciones registrada en este estudio no debe subestimarse. Se requiere el adiestramiento adecuado en la ejecución del tratamiento de pacientes con litiasis complejas.

Este estudio representa una base de datos importante, por la cantidad de pacientes analizados, incluso puede funcionar como precedente para efectuar investigaciones futuras, que consideren variables como: tasa libre de cálculos y complicaciones por subgrupos.

\section{CONCLUSIONES}

La nefrolitotomía percutánea es una técnica quirúrgica efectiva en el tratamiento de la litiasis renal, que supone menor índice de complicaciones. Los resultados obtenidos en este ensayo coinciden con los reportados en el estudio CROES.

\section{REFERENCIAS}

1. Mc Dougal WS, et al. Campbell-Walsh Urology, $10^{\text {th }}$ ed. Amsterdam: Elsevier, 2012.

2. Türk C, et al. EAU Guidelines on urolithiasis. European Association of Urology 2015. Dirección URL: <https:// uroweb.org/wp-content/uploads/EAU-Guidelines-Urolithiasis-2016-1.pdf >.

3. Preminger GM, et al. Chapter 1: AUA guideline on management of staghorn calculi: diagnosis and treatment recommendations. J Urol 2005;173(6):1991-2000.

4. Fernström I, Johansson B. Percutaneous pyelolithotomy. A new extraction technique. Scand J Urol Nephrol 1976;10:257-259.

5. Seitz $\mathrm{C}$, et al. Incidence, prevention, and management of complications following percutaneous nephrolitholapaxy. Eur Urol 2012;61(1):146-58. DOI: 10.1016/j.eururo.2011.09.016

6. Labate $G$, et al. The percutaneous nephrolithotomy global study: classification of complications. J Endourol 2011;25(8):1275-1280. DOI: 10.1089/end.2011.0067

7. de la Rosette JJ, et al. Categorisation of complications and validation of the clavien score for percutaneous nephrolithotomy. Eur Urol 2012;62(2):246-55. DOI: 10.1016/j. eururo.2012.03.055

8. Tefekli A, et al. Classification of percutaneous nephrolithotomy complications using the modified clavien grading system: looking for a standard. Eur Urol 2008;53:184-190. DOI: 10.1016/j.eururo.2007.06.049 
Trujillo-Ríos K y col. Éxito y morbilidad de la nefrolitotomía percutánea

9. Dindo D, Demartines N, Clavien PA. Classification of surgical complications: a new proposal with evaluation in a cohort of 6336 patients and results of a survey. Ann Surg 2004;240:205-213.

10. de la Rosette JJ, et al. The Clinical Research Office of the Endourological Society (CROES) Percutaneous Nephrolitho- tomy (PCNL) Global Study: indications, complications and outcomes in 5803 patients. J Endourol 2011;25(1):11-17. DOI: 10.1089/end.2010.0424

11. Deters LA, et al. Evaluating the definition of "stone free status" in contemporary urologic literature. Clin Nephrol 2011;76:354-7.

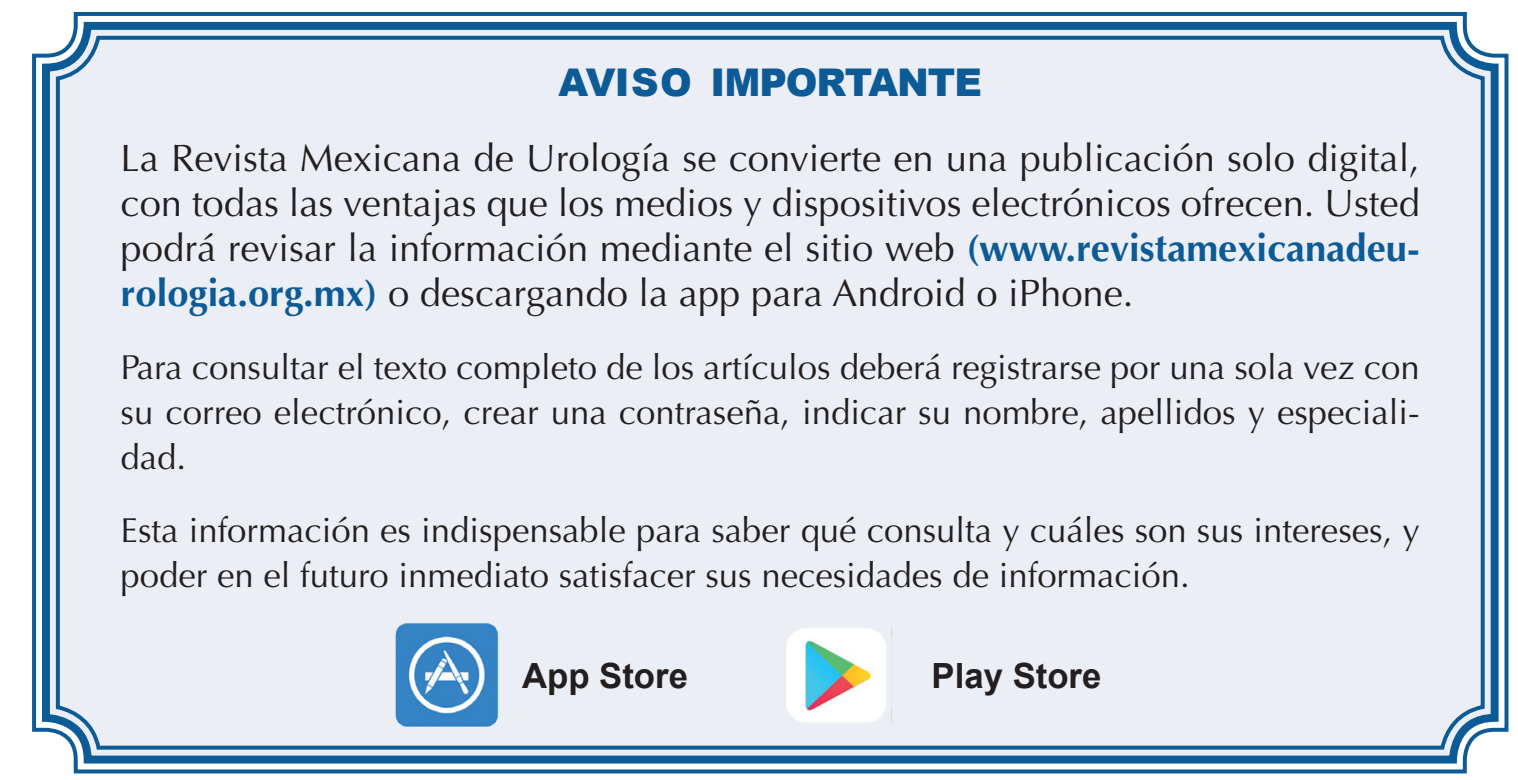

